\title{
Computer-assisted identification of the gingival sulcus and periodontal epithelial junction on high-frequency ultrasound images
}

\author{
Radu Chifor ${ }^{1}$, Mindra Eugenia Badea1, Delia-Alexandrina Mitrea², Iulia Clara Badea1, \\ Maria Crisan ${ }^{3}$, Ioana Chifor ${ }^{1}$, Ramona Avram ${ }^{1}$
}

${ }^{1}$ Department of Preventive Dentistry, "Iuliu Hatieganu" University of Medicine and Pharmacy, ${ }^{2}$ Computer Science Department, Technical University, ${ }^{3}$ Department of Histology/Dermatology, "Iuliu Hatieganu" University of Medicine and Pharmacy, Cluj-Napoca, Romania

\begin{abstract}
The primary aim of this study was to demonstrate that periodontal ultrasonography is a reliable method with which to identify and evaluate the attachment level of the gingival junctional epithelium. A secondary aim was to devise an automated computer-assisted method that allows the examiner to more easily identify the gingival sulcus contour on ultrasound images. Material and methods: This in vitro study was carried out on 36 sites on the lingual surface of eight pig mandibles. For each site, periodontal ultrasonography was performed by the same examiner, using DermaScan C Cortex Technology (Denmark) with a $20-\mathrm{MHz}$ transducer. Subsequently, the mandibles were sectioned with a microtome and examined by direct microscopy. To facilitate identification of the gingival sulcus on ultrasound images, a computational method was adopted. Results: Computer processing of the ultrasound images slightly modified the contour of the gingival sulcus. The absolute mean differences in the linear measurements of the Dermascan-automated computer-generated values and the corresponding values of microscopy, which is the gold standard, varied between 0.06 and $1.75 \mathrm{~mm}$. Statistical analysis showed that with respect to the gingival sulcus height, the correlation between the computer-processed ultrasound images and the direct microscopy images was stronger than the correlation between the non-processed ultrasound images and those from direct microscopy. Conclusions: Ultrasonographic examination of the periodontal tissues allows the examiner to localize the gingival epithelial attachment level and provides substantial data regarding the soft gingival tissues.
\end{abstract}

Keywords: junctional epithelium, high frequency ultrasound imaging, periodontal ultrasonography, periodontal diagnosis

\section{Introduction}

Periodontal disease, with all its stages of evolution, is extremely widespread in the adult population [1]. Gingival bleeding is highly prevalent and advanced stages of periodontal disease, with deep periodontal pockets $(\geq 6$ $\mathrm{mm}$ ), is seen in $10-15 \%$ of adults worldwide [1].

The anatomy of the marginal periodontium is complex. At the bottom of the gingival sulcus lies the epi-

Received 26.03.2015 Accepted 02.05.2015

Med Ultrason

2015, Vol. 17, No 3, 273-279

Corresponding author: Ioana Chifor

15 , Victor Babes str

400012 Cluj-Napoca Romania

Tel: +40 744195229

Email: ioana_chi@yahoo.com thelial insertion of the gingiva on the tooth surface, also called the junctional epithelium. The junctional epithelium plays a key role in the evolution of periodontal disease. The diagnosis and monitoring of periodontal disease evolution are performed according to this epithelial insertion. Clinical periodontal probing does not return a depth equal with the histological one [2]. Damage to the epithelial junction results in its inability to protect the periodontal space, marking the onset of periodontal disease [3]. The margin of the gum has an extremely important role in protecting the tissues that support the teeth, but also dental implants. If this margin loses its adhesion, through epithelial insertion, then oral health is adversely affected and periodontal disease develops [4]. The first effects of an infection with Porphyromonas gingivalis occur at the level of the epithelial junction, through al- 
terations in keratinization, the degree of desquamation, and mitotic activity [5]. The concentration of $P$. gingivalis is directly related to the onset of the destruction of the epithelial barrier [6]. In response to the increased forces (such as orthodontic forces) on the teeth, the epithelial insertion becomes modified, setting off an immunological response in addition to inflammation [7].

The junctional epithelium and the connective tissue together form a protective barrier between the deep periodontal tissues and the oral environment. These two dimensions together form the biological width. The biological width is important in designing dental prostheses and in establishing the diagnosis for periodontal diseases, and must therefore be measured with high precision. In individuals with a dental prosthesis, the biological width must always be respected as the periodontal system reacts according to the contact area of the prosthesis with the marginal periodontium $[8,9]$.

The biological width and the epithelial junction length have been very accurately measured only on autopsy specimens, examining tissue sections under a microscope $[8,10,11]$. In dental patients, clinical and radiological examinations are used to approximate the position of the epithelial insertion [2,9].

The main periodontal structures can be evaluated by periodontal ultrasonography (US). For example, in the evaluation of the alveolar crest, US was used to estimate alveolar bone levels to monitor horizontal and vertical bone resoption [12-14]. The gingival soft tissues have been examined using $40-\mathrm{MHz}$ periodontal ultrasound imaging, which included measurements of gingival thickness $[15,16]$. However, the transducers needed for this purpose are usually very large, and accordingly, examination of the interdental area is difficult. With the development of ultrasonic periodontal probes, interdental areas can be included in the examination. In fact, ultrasonic periodontal probes are able to replace manual periodontal probing [17-19].

The primary aim of this study was to demonstrate that periodontal ultrasonography offers a reliable method for identifying and evaluating the attachment level of the gingival junctional epithelium and the gingival biological width. A secondary aim was to devise an automated computer-assisted method that allows the examiner to more easily identify the gingival sulcus contour.

\section{Material and methods}

This in vitro study was carried out on 36 sites on the lingual surface of eight mandibles from healthy young pigs (age 10-12 months) with a body mass of $100-110$ $\mathrm{kg}$. The inclusion criterion was the teeth with healthy periodontal tissue with no lesions. The pigs were raised and slaughtered for alimentary purposes in farms organized according to European Union legislation and were not experimental animals. Therefore, the Medical Ethics Commission of the University of Medicine and Pharmacy of Cluj-Napoca, Romania, where the study was conducted, waived the need for approval of the study (document no. 5/16.01.2014).

\section{Periodontal US}

Gutta percha cones were glued onto the teeth at every examination site, that is, on the lingual surface, parallel to the longitudinal axis of the tooth, without touching the periodontal tissue. A periodontal US examination was then performed at each site indicated by the glued gutta percha landmarks. Periodontal ultrasonography was carried out using DermaScan C Cortex Technology (Denmark). The $20-\mathrm{MHz}$ two-dimensional transducer was positioned directly on the alveolar process, parallel to the longitudinal axis of the tooth. Ultrasonographic images were then recorded on the lingual surface of the alveolar process.

\section{Section preparation}

Immediately after US examination, the mandibles were sectioned with a microtome low-speed saw (Buehler Isomet, Lake Bluff, Illinois, USA). The sections were cut exactly through the gutta percha landmarks, parallel to the longitudinal axis of the tooth (fig 1). Longitudinal sections in the frontal plane were thus obtained.

\section{Direct microscopy}

Direct microscopy of the sections was performed using an Olympus CKX41 microscope (Olympus, Waltham, Massachusetts, USA) at $4 \times$ magnification, without refrigeration or further preparation of the tissue sections.

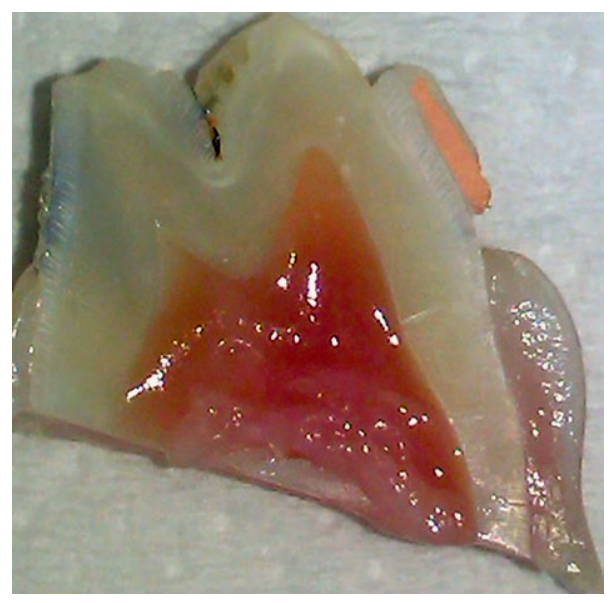

Fig 1. Longitudinal section through a swine tooth. The gutta percha landmarks are glued to the tooth. 


\section{Computer-assisted identification of the gingival sulcus}

On the US images the examiner drew the gingival sulcus contour.

To facilitate identification of the gingival sulcus on US images with the gingival contour marked, a methodology comprising the following steps was adopted (fig $2)$ : 1) The original image was converted to gray scale; 2) The k-means clustering method for image segmentation was employed to discover groups of pixels having similar intensity levels and to better highlight the anatomic elements of the image; 3) Active contour models ("snakes") were used for contour detection before and after step 2, to detect the gingival sulcus epithelium contour; the two methods of contour detection implementation were then compared; 4) Adaptive thresholding, using the minimum Euclidean distance, computed between the original and the target image [15], was performed on the images obtained in step 2 to obtain the black-and-white version of the image; the final purpose was an improved visualization of the anatomic elements within the image; 5) Edge detection using both the horizontal and vertical Sobel convolution kernels (averaged) [20] was carried out on the image obtained at step 1, to better highlight the margins and contours of the main anatomic structures on the image. Both the k-means clustering method and active contour models were implemented in Matlab; edge detection and adaptive thresholding methods were implemented in Visual $\mathrm{C}++$.

The region corresponding to the gingival sulcus consists of pixels having various image intensities of small values. Thus, the active contour model applied to this area of non-uniform color is directed at local minima. In k-means clustering, groups (classes) of pixels similar in intensity are assembled, labeling all corresponding pixels with the same value (the class label) such that the risk of local minima detection is reduced. Ultimately, the aim

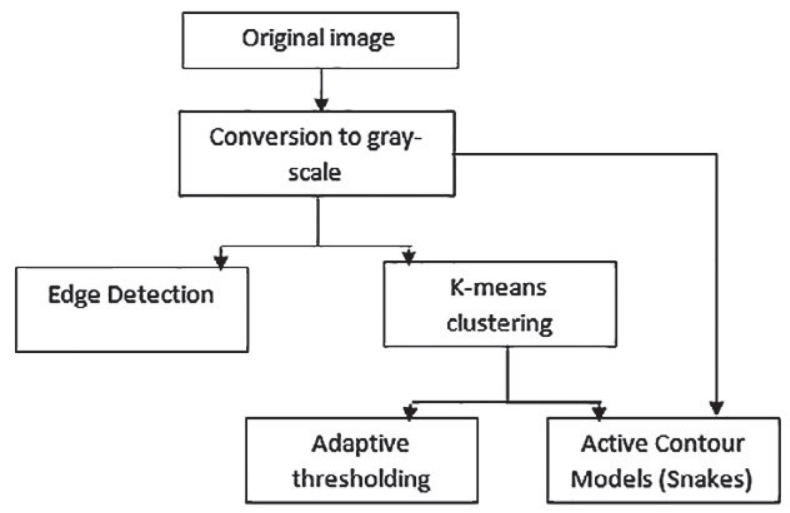

Fig 2. The flow of the image processing operations. of the active contour models is to provide a highly accurate identification of the gingival sulcus contour, even on noisy or low-quality US images, when this contour is difficult to detect by a clinician, even an experienced one. The methods of adaptive thresholding and edge detection are applied in parallel, after conversion of the image to gray scale, to better highlight the corresponding anatomic structures.

After image processing, the method of two-dimensional active contour models ("snakes") was used to detect the contours of the anatomic structures. This powerful contour detection method is robust to noise in the image and is based on energy minimization. It was considered appropriate in the detection of the gingival sulcus epithelium because the corresponding contour is difficult to detect by the human eye or by using classical edge and contour detection methods [21,22].

\section{Statistical analysis}

Statistical analysis was performed using SPSS $^{\circledR}$ V.18.0 (SPSS Inc., Chicago, IL, USA) and Microsoft Excel 2010. Descriptive statistics were calculated, and values are given as means \pm standard deviation (SD). A Spearman correlation index was used for correlations between Dermascan, microscopy (as gold standard), and computer-generated measurement. All variables were continuous and had a normal distribution. For statistical validation of the data obtained by measurements, a two-tailed Student's t-test was employed to compare the means of variables. Significance was defined at a $p$ value $<0.05$.

\section{Results}

All mandibles could be examined as planned and the microtome sections were made exactly on the gutta percha landmarks, which stayed in place after sectioning.

The following anatomic elements were identified on the ultrasound images: enamel, tooth root, gingival sulcus, alveolar crest, cortical bone, attached gingival tissue, and free gingival margin (fig 3). The enamel, cementum, and cortical bone are hyperechogenic areas, with the enamel brighter than the cementum. The connective tissue is a darker hypoechogenic area. The gingival sulcus is a hypoechogenic area well delimited by the tooth and by the free gingival margin. The following distances were measured using the Dermascan $\mathrm{C}$ software: the height of the gingival sulcus (D-HGS), the distance between the alveolar crest and cementum-enamel junction (D-ACCEJ), and the width of the periodontal space (D-PSW).

The same elements identified at US examination were visualized by direct microscopy: enamel, tooth root, gingival sulcus, alveolar crest, cortical bone, attached gingi- 
a)
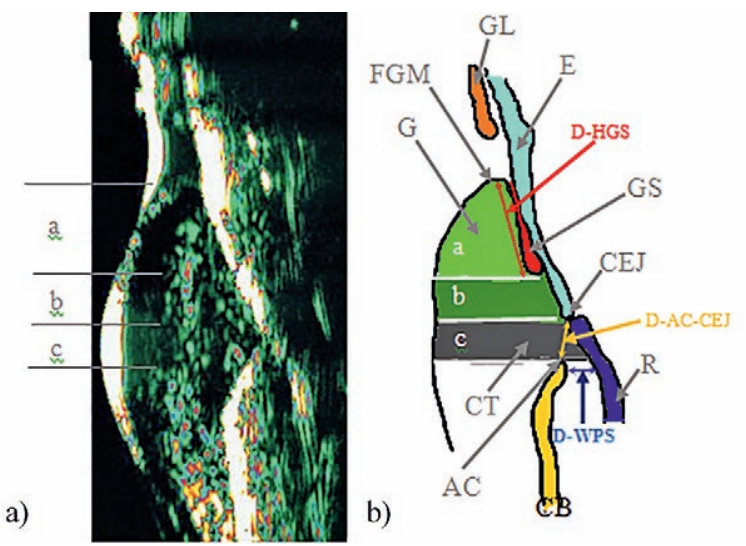

Fig 3. Periodontal ultrasonography using 20-MHz DermaScan C Cortex Technology a) periodontal ultrasonography using 20-MHZ DermaScan C Cortex Technology; b) explanatory drawing: gutta percha landmark (GL), enamel tooth crown (E), gingival sulcus (GS), cementum-enamel junction (CEJ), tooth root $(\mathrm{R})$, aleveolar crest (AC), connective tissue (CT), gingival tissue $(\mathrm{G})$, free gingival margin (FGM), cortical bone (CB); ahistological sulcus, b- epithelial attachment, c- connective tissue attachment, biological width $(b+c)$.
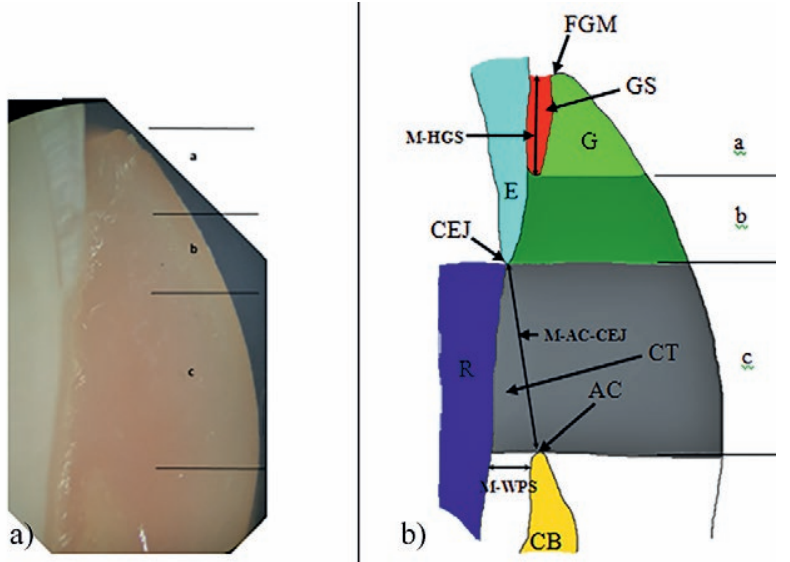

Fig 4. Direct microscopy ( $4 \times$ magnification): a) periodontal direct microscopy using an Olympus CKX41 microscope, magnification $4 \times$; b) explanatory drawing: enamel tooth crown (E), gingival sulcus (GS), cementum-enamel junction (CEJ), tooth root $(\mathrm{R})$, aleveolar crest (AC), connective tissue (CT), gingival tissue $(\mathrm{G})$, free gingival margin (FGM), cortical bone (CB); ahistological sulcus, b- epithelial attachment, c- connective tissue attachment, biological width $(b+c)$.

val tissue, and free gingival margin (fig 4). The following distances were measured using the Olympus CKX41 software: the height of the gingival sulcus (M-HGS), the distance between the alveolar crest and cementum-enamel junction (M-AC-CEJ), and the width of the periodontal space (M-PSW).

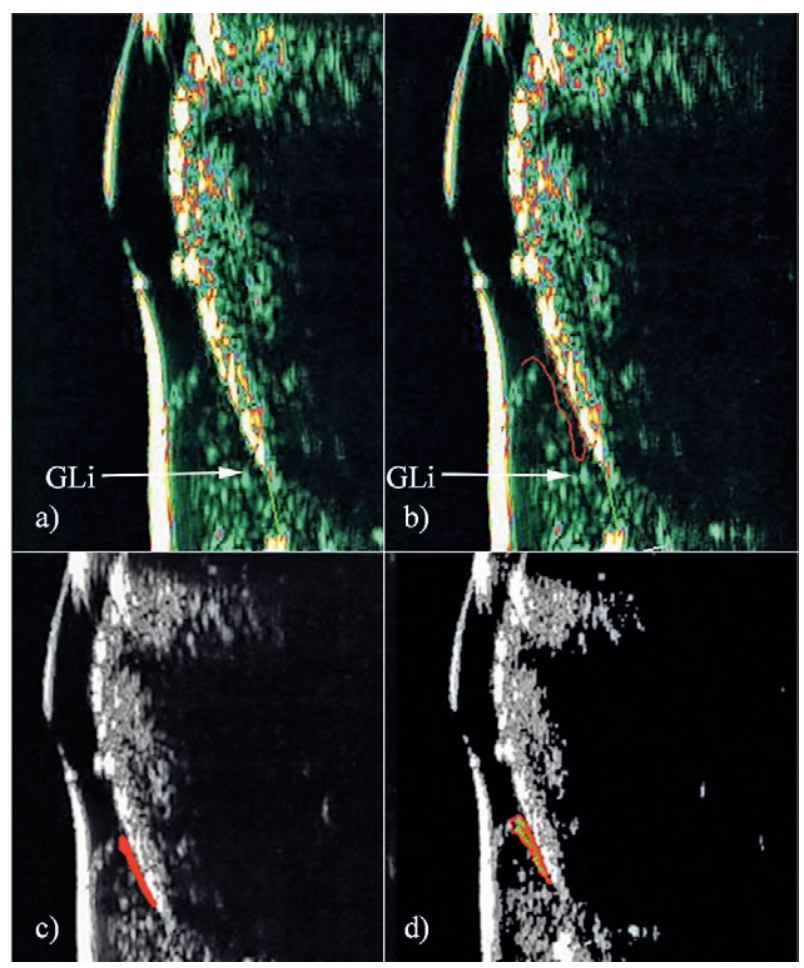

Fig 5. Periodontal ultrasonography using 20-MHz DermaScan $\mathrm{C}$ Cortex Technology. Computer generation of a gingival sulcus contour: a) Periodontal ultrasonography using 20-MHz DermaScan C Cortex Technology without gingival contour. The gingival ligaments are attached to the tooth (GLi); b) The real contour as traced by the medical expert; c) The final contour detected on the gray-scale image and d) after the application of $\mathrm{k}$-means clustering $(\mathrm{k}=4)$.

The contour is initialized by the user and then automatically evolves towards the real margins of the anatomic structure (gingival sulcus), which according to the "snakes" algorithm respects the condition of minimum energy.

Figure 5 depicts the final contour obtained through the "snakes" algorithm. The contour was determined directly from the gray-scale image, as well as from the image obtained after the application of the k-means clustering algorithm $(\mathrm{k}=4)$.

To obtain the computer-generated measurements, of the height of the gingival sulcus (C-HGS), the experiments were performed on 36 ultrasound images of $150 \times$ 150 pixels. The $\mathrm{k}$-means clustering method was tested for $\mathrm{k}$ parameter values of $2,3,4$, and 5 , with the best results being those for 3 and 4 .

For the active contour models, weights of 0.1, 0.09, 0.12 , and 2 were assigned to the elastic energy, the bending energy, the balloon force, and the external energy, re- 
spectively, as they provided the best results. This method was employed before and after the application of k-means clustering, with the best results achieved after application of the unsupervised classification algorithm. The results obtained for segmentation through active contour models were validated by their comparisons with the contours traced manually by the medical expert.

After computer-assisted identification of the gingival sulcus, the gingival sulcus height was measured on computer processed ultrasound images (C-GSH).

There was a strong correlation between D-AC-CEJ and M-AC-CEJ, and between D-PSW and M-PSW with $\mathrm{R}$ coefficient $=0.854(85 \%)$, respectively $\mathrm{R}=0.87(87 \%)$. The correlation between M-GSH and D-GSH measurements was lower $\mathrm{R}=0.557$ (55.7\%). The correlation between M-GSH which represented the gold standard and C-GSH was higher than the one obtained before the application of the computer algoritm with an $\mathrm{R}$ coefficient $=0.78(78 \%)$.

Tables I and II list the mean values of the paired statistics. The absolute mean differences of the linear measurements obtained by Dermacan and the Dermascan-automated computer-generated values, and the corresponding values obtained by microscopy, as the gold standard, varied between 0.06 and $1.75 \mathrm{~mm}$. The absolute values of the $\mathrm{SD}$ of these differences ranged between 0.09 and 1.75. Significant correlations $(p<0.05)$ were found between the values measured by all three methods (Dermascan, computer, and microscopy).

\section{Discussions}

The biological width of the attached gingival tissue is extremely important in diagnosing and tracking the evolution of periodontal diseases. The biological width is bordered by the bottom of the gingival sulcus and the cortical bone (alveolar crest). The strong correlation between D-AC-CEJ and M-AC-CEJ, and between D-PSW and M-PSW showed that cortical bone is easily identifiable on US images. As such, attention could then be focused on identifying the gingival sulcus, the limits of which are often difficult to determine on US images. The weakest correlation was between D-GSH and M-GSH $(\mathrm{R}=0.557)$, which justified computer processing of the US images. Automated processing slightly modified the contour of the gingival sulcus. Statistical analysis showed a stronger correlation between C-GSH and M-GSH than between D-GSH and M-GSH, demonstrating that the new contour of the gingival sulcus was closer to the histological contour determined on the gold-standard microscopy images. The other two dimensions, D-AC-CEJ and D-WPS, or M-AC-CEJ and M-WPS, were measured because they are the ones traditionally used in assessing horizontal and vertical bone atrophy during periodontal diagnosis.

Both k-means clustering and active contour models ("snakes") have been implemented in other medical image segmentation applications [23-25]. However, these techniques have not been used for segmentation tasks involving periodontal high-frequency ultrasound images.

To monitor periodontal evolution following treatment, clinical parameters are often obtained by manual probing [26]. The patient is followed over a short time span (weeks, months) such that the development of minor changes, which could be important in the long term, are extremely difficult to detect. Moreover, the findings of these examinations are influenced by the type and size of the periodontal probes used [27] and by the pressure exerted on the anatomic structure by the operator. Thus, for these types of studies, as well as those in which orthodontic treatment is monitored, i.e., where minor changes in

Table I. Paired statistics of the Dermascan and microscopy (gold standard) measurements

\begin{tabular}{llllll}
\hline & & \multicolumn{2}{c}{ Differences between microscopy and Dermascan measurements } \\
Periodontium variables & Mean difference & SD & R & Significance (two-tailed) \\
\hline Values in mm & M-AC-CEJ - D-AC-CEJ & -1.748 & 1.638 & 0.854 & $\mathrm{p}=0.002$ \\
& M-PSW - D-PSW & -0.061 & 0.087 & 0.772 & $\mathrm{p}<0.001$ \\
& M-GSH - D-GSH & -0.258 & 0.453 & 0.557 & $\mathrm{p}=0.001$ \\
\hline
\end{tabular}

D-AC-CEJ, Dermascan distance between the alveolar crest and cementum-enamel junction; D-GSH, Dermascan gingival sulcus height; D-PSW, Dermascan periodontal space width; M-AC-CEJ, microscopy distance between the alveolar crest and cementum-enamel junction; M-GSH, microscopy gingival sulcus height; M-PSW, microscopy periodontal space width; R, correlation coefficient; SD standard deviation.

Table II. Paired statistics of the computer-generated values and the microscopy (gold-standard) measurements.

\begin{tabular}{llllll}
\hline & & \multicolumn{4}{l}{ Differences between the microscopy and the computer-generated values } \\
Periodontium variable & Mean difference & SD & R & Significance (two-tailed) \\
\hline Value in $\mathrm{mm}$ & M-GSH - C-GSH & -0.181 & 0.322 & 0.785 & $\mathrm{p}=0.002$ \\
\hline
\end{tabular}

C-GSH, computer-generated gingival sulcus height; M-GSH, microscopy gingival sulcus height; R, correlation coefficient; SD standard deviation. 
gingival attachment could predict negative consequences, assessment of the periodontal tissue with a high accuracy imaging method is very useful for diagnosis purposes. In their literature review, Marotti et al [28] concluded that periodontal US is gaining increasing acceptance because of the accuracy of the data and because it is a non-invasive, painless, and inexpensive method. Moreover, it does not involve the use of ionizing radiation, unlike radiological examination of the periodontium, with exposure of both patients and the medical staff. In addition, radiological methods do not provide information on the soft tissues, such as the fixed gum and free gingival margins. Both X-rays and cone beam computed tomography yield information only on vertical and horizontal bone atrophies. While cone beam computed tomography provides more information than periapical X-rays [29], it cannot be used for regular periodontal checkups because of the high doses of radiation required for a proper evaluation.

The main disadvantage of US is that, in many situations, the images are difficult to interpret and certain areas in the oral cavity are hard to examine because of the large size of the transducers. An additional drawback occurs when interpreting US images of the marginal periodontal area, because the differences in the echogenicities of the different tissues are small, and as such identification of the gingival sulcus is difficult.

Until recently, US of periodontal tissues was used mainly in an experimental setting. Zimbrean et al examined periodontal tissues at $40 \mathrm{MHz}$ and were able to measure dimensions related to gingival, dental, and bone tissues with high accuracy [30]. Mahmoud et al, in an in vitro study, successfully obtained three-dimensional US reconstructions of mandible bone defects [31]. Tsiolis et al concluded, after a study performed on pig mandibles with a $20-\mathrm{MHz}$ transducer, that US is a highly accurate and repeatable technique for periodontal assessment and can be used to image the main periodontal structures [12].

The novelty of our study lies in the identification and visualization on US images of the gingival sulcus, without the need for any other element that could influence the gingival attachment level. Moreover, the computer algorithm used in the analysis significantly increased the accuracy of the measurements, as confirmed by the fact that the values did not significantly differ from those obtained by microscopy. Thus, the gingival tissues and cortical bone position with respect to hard dental tissues can be assessed very accurately, improving the precision and quality of periodontal diagnosis and treatment monitoring.

The computerized method can be further optimized by also considering textural features, which will provide better separation between the gingival sulcus region and neighboring regions, with enhanced accuracy of the method based on active contour models. With further development, it can be used even by clinicians with less experience in the field of ultrasonography.

The limits of the study consist of restricting the examined area at the lingual surface. The buccal one was impossible to be examined due to the high level of muscle insertions, resulting in a surface of the alveolar process smaller than the transducer footprint. Due to the size of the trasducer and the low penetrability it was impossible to examine human patients, therefore the study was performed only on an animal experimental model. Further research using devices with different size transducers is necessary to allow application of the method in humans, with the appropriate size transducer chosen according to the difficulty and the area of the examination. Studies performed with devices having variable frequencies $(\mathrm{MHz})$ would also be of interest, selecting the most appropriate frequency for visualizing the periodontal tissues, in order to obtain a high resolution of the images and to have a penetrability which allows us to do the examinations.

\section{Conclusions}

Periodontal US allows precise examination and localization of the gingival epithelial attachment level, while providing substantial data on the soft gingival tissues. Vertical and horizontal bone resorption can be evaluated, which is extremely important in the diagnosis and monitoring of periodontal diseases. Periodontal US was further improved by the computerized processing of the recorded ultrasound images.

\section{Acknowledgments}

We would like to express our gratitude to Cortex Technology for lending us the DermaScan C and its 20$\mathrm{MHz}$ two-dimensional transducer device used in this study. The work is part of the $\mathrm{PhD}$ scholarship project POSDRU 78702.

The contribution of the author from the Technical University of Cluj-Napoca was supported by the Post-Doctoral Programme POSDRU/159/1.5/S/137516, a project cofunded by the European Social Fund through the Human Resources Sectorial Operational Program 2007-2013.

Conflict of interests: none

\section{References}

1. Petersen PE, Ogawa H. Strengthening the prevention of periodontal disease: the WHO approach. J Periodontol 2005; 76: 2187-2193. 
2. Newman MG, Takei HH, Klokkevold PR, Carranza FA. Carranza's Clinical Periodontology. Tenth Edition. Edited by Saunders Elsevier, 2006: 16-26, 1224.

3. Nanci A, Bosshardt DD. Structure of periodontal tissues in health and disease. Periodontol 2000 2006; 40: 11-28.

4. Larjava H, Koivisto L, Häkkinen L, Heino J. Epithelial integrins with special reference to oral epithelia. J Dent Res 2011; 90: 1367-1376.

5. Yilmaz O. The chronicles of Porphyromonas gingivalis: the microbium, the human oral epithelium and their interplay. Microbiology 2008. 154: 2897-2903.

6. Katz J, Sambandam V, Wu JH, Michalek SM, Balkovetz DF. Characterization of Porphyromonas gingivalis-Induced Degradation of Epithelial Cell Junctional Complexes. Infect Immun 2000; 68: 1441-1449.

7. Tadokoro O, Kawahara I, Vandevska-Radunovic V. Reactions of periodontal ligament epithelial cell clusters and OX6-immunopositive cells to experimental tooth movement and periodontitis. J Periodontal Res 2011; 46: 584-591.

8. Xie GY, Chen JH, Wang H, Wang YJ. Morphological measurement of biologic width in Chinese people. J Oral Sci 2007; 49: 197-200.

9. Nugala B, Kumar BS, Sahitya S, Krishna PM. Biologic width and its importance in periodontal and restorative dentistry. J Conserv Dent 2012; 15: 12-17.

10. Vacek JS, Gher ME, Assad DA, Richardson AC, Giambarresi LI. The dimensions of the human dentogingival junction. Int J Periodontics Restorative Dent 1994; 14: 154-165.

11. Alpiste-Illueca F. Morphology and dimensions of the dentogingival unit in the altered passive eruption. Med Oral Patol Oral Cir Bucal 2012; 17: 814-820.

12. Tsiolis FI, Needleman IG, Griffiths GS. Periodontal ultrasonography. J Clin Periodontol 2003; 30: 849-854.

13. Spranger H. Ultra-sonic diagnosis of marginal periodontal diseases. Int Dent J 1971; 21: 442-455.

14. Ursell MJ. Relationships between alveolar bone levels measured at surgery, estimated by transgingival probing and clinical attachment level measurements. J Clin Periodontol 1989; 16: 81-86.

15. Farr C. Ultrasonic probing: The wave of the future in dentistry. Dent Today 2000; 19: 86-91.

16. McCombs GB, Hinders M. The potential of the ultrasonic probe. Dimensions Dent Hyg 2006; 4: 16-18.

17. Hou J, Rose ST, Hinders MK. Ultrasonic periodontal probing based on dynamic wavelet fingerprint. EURSASIP J Appl Signal Processing 2005; 7: 1137-1146.
18. Muller HP, Stahl M, Eger T. Dynamics of mucosal dimensions after root coverage with a bioresorbable membrane. $\mathrm{J}$ Clin Periodontol 2000; 27: 1-8.

19. Muller HP, Schaller N, Eger T, Heinecke A. Thickness of masticatory mucosa. J Clin Periodontol 2000; 27: 431-436.

20. Toennies KD. Guide to medical image analysis. Methods and algorithms. Springer Verlag, London 2012: 111-146.

21. Witten IH, Frank E. Data Mining: Practical Machine Learning Tools and Techniques. (2nd edition). Morgan Kaufmann, San Francisco 2005: 35-80.

22. McIntosh C, Hamarneh G. Medical Image Segmentation: Energy Minimization and Deformable Models (Chapter 23). In: Farncombe T, Iniewski K. (Eds.). Medical Imaging: Technology and Applications. 2013: 661-692.

23. Mohd Khuzi A, Besar R, Wan Zaki W, Ahmad N. Identification of masses in digital mammogram using gray level co-occurrence matrices. Biomed Imaging Interv J 2009; 5: 17-30.

24. Sassi O, Sellami L, Ben Slima M, Chtourou K, Hamida AB. Improved spatial gray level dependence matrices for texture analysis. International Journal of Computer Science \& Information Technology (IJCSIT) 2012; 4: 209-219.

25. Chai HY, Wee LK, Swee TT, et al. Gray-level co-occurrence matrix bone fracture detection. Am J Applied Sci 2011; 8: 26-32.

26. Berakdar M, Callaway A, Eddin MF, Ross A, Willershausen B. Comparison between scaling-root-planing (SRP) and SRP/photodynamic therapy: six-month study. Head Face Med 2012; 8: 12.

27. Holtfreter B, Alte D, Schwahn C, Desvarieux M, Kocher T. Effects of different manual periodontal probes on periodontal measurement. J Clin Periodontol 2012; 39: 1032-1041.

28. Marotti J, Heger S, Tinschert J, et al. Recent advances of ultrasound imaging in dentistry - a review of the literature, Oral Surg Oral Med Oral Pathol Oral Radiol 2013; 115: 819-832.

29. Mohan R, Mark R, Sing I, Jain A. Diagnostic Accuracy of CBCT for Aggressive Periodontitis. J Clin Imaging Sci 2014; 4(Suppl 2): 2.

30. Zimbran A, Dudea S, Dudea D. Evaluation of periodontal tissues using $40 \mathrm{MHz}$ ultrasonography. Preliminary report. Med Ultrason 2013; 15: 6-9.

31. Mahmoud AM, Ngan P, Crout R, Mukdadi OM. High-resolution 3D ultrasound jawbone surface imaging for diagnosis of periodontal bony defects: an in vitro study. Ann Biomed Eng 2010; 38: 3409-3422. 